\title{
aPROMISE: A Novel Automated-PROMISE platform to Standardize Evaluation of Tumor Burden in 18F-DCFPyL (PSMA) images of Veterans with Prostate Cancer
}

\author{
Authors: Nicholas Nickols ${ }^{1,2,3,4}$, Aseem Anand ${ }^{5,6,7}$, Kerstin Johnsson $^{7}$, Johan Brynolfsson ${ }^{7}$, Pablo Borreli ${ }^{8}$, \\ Neil Parikh², Jesus Juarez ${ }^{2}$, Lida Jafari ${ }^{9}$, Mattias Eiber ${ }^{10}$, Matthew Rettig ${ }^{2,3,11}$.
}

\begin{abstract}
${ }^{1}$ Radiation Oncology Service, VA Greater Los Angeles Healthcare System, Los Angeles, CA; ${ }^{2}$ Department of Radiation Oncology, David Geffen School of Medicine, University of California Los Angeles; Los Angeles, CA; ${ }^{3}$ Department of Urology, David Geffen School of Medicine, University of California Los Angeles; Los Angeles, CA; ${ }^{4}$ Institute of Urologic Oncology, Jonsson Comprehensive Cancer Center, University of California Los Angeles; Los Angeles, CA; ${ }^{5}$ Department of Translational Medicine, Division of Urological Cancer, Lund University, Malmö, Sweden; ${ }^{6}$ Department of Medicine, Memorial Sloan Kettering Cancer Center, New York, USA; ${ }^{7}$ Department of Data Science and Machine Learning, EXINI Diagnostic AB, Lund, Sweden; ${ }^{8}$ Department of Clinical Physiology, Region Vastra Gotland, Sahlgrenska University Hospital, Gothenburg, Sweden; 'Imaging Service, VA Greater Los Angeles Healthcare System; Los Angeles, CA; ${ }^{10}$ Department of Nuclear Medicine, Klinikum Rechts der Isar, Technical University of Munich, Munich, Germany; ${ }^{11}$ Division of Hematology-Oncology, VA Greater Los Angeles Healthcare System.
\end{abstract}

\section{Corresponding Author:}

\section{Matthew Rettig, MD}

Professor of Medicine and Urology, Medical Director, Prostate Cancer Program

Institute of Urologic Oncology, Associate Director, Hematology-Oncology Fellowship Program,

David Geffen School of Medicine at UCLA

Chief, Division of Hematology-Oncology

VA Greater Los Angeles - West Los Angeles

E-mail: mrettig@mednet.ucla.edu

Phone: +13102683622

\section{Word Count: $\mathbf{5 0 0 0}$ words MAX}

Running Title: aPROMISE standardized PSMA analysis 


\section{ABSTRACT}

Rationale: Standardized staging and quantitative reporting is necessary to demonstrate the association of ${ }^{18} \mathrm{~F}-\mathrm{DCFPyL}$ PET/CT (PSMA) imaging with clinical outcome. This work introduces an automated platform to implement and extend the Prostate Cancer Molecular Imaging Standardized Evaluation (PROMISE) criteria aPROMISE. The objective is to validate the performance of aPROMISE in staging and quantifying disease burden in patients with prostate cancer who undergo PSMA Imaging.

Methods: This was a retrospective analysis of 109 Veterans with intermediate and high-risk prostate cancer, who underwent PSMA imaging. To validate the performance of aPROMISE, two independent nuclear-medicine physicians conducted aPROMISEassisted reads, resulting in standardized reports that quantify individual lesions and stage the patients. Patients were staged as having local only disease (miNOMO); regional lymph node only (miN1M0), metastatic disease only (miN0M1), and with both regional and distant metastatic disease (miN1M1). The staging obtained from aPROMISE-assisted reads was compared with the staging by conventional imaging. Cohen's pairwise kappa agreement was used to evaluate the inter-reader variability. Correlation coefficient and ICC was used to evaluate the inter-reader variability of the quantitative assessment (miPSMA-index) in each stage. Kendall Tau and t-test was used to evaluate the association of miPSMA-index with PSA and Gleason Score.

Results: All PSMA images of 109 veterans met the DICOM conformity and the requirements for the aPROMISE analysis. Both independent aPROMISE-assisted analyses demonstrated significant upstaging in patients with localized $(23 \% ; \mathrm{N}=20 / 87)$ and regional tumor burden $(25 \% ; \mathrm{N}=2 / 8)$. However, a significant number of patients with 
bone metastases identified on conventional imaging (NaF PET/CT) were downstaged $(29 \% ; \mathrm{N}=4 / 14)$. The comparison of the two independent aPROMISE-assisted reads demonstrated a high kappa agreement - 0.82 (miN0M0), 0.90 (miN1M0), and 0.77 (miN0M1). The Spearman correlation of quantitative miPSMA-index was 0.93, 0.96 and 0.97, respectively. As a continuous variable, miPSMA index in the prostate (miT) was associated with risk groups defined by the PSA and Gleason..

Conclusion: Here we demonstrate consistency of the aPROMISE platform between readers and observed substantial upstaging in PSMA imaging compared to the conventional imaging. aPROMISE may contribute to the broader standardization of PSMA imaging assessment and to its clinical utility in management of prostate cancer patients.

Key Words: ${ }^{18} \mathrm{~F}-\mathrm{DCFPyL} ; \quad \mathrm{PSMA}$; aPROMISE; segmentation; quantification; standardization; Prostate Cancer 


\section{INTRODUCTION}

Prostate cancer is the most common solid tumor in men, with nearly 192,000 incidence cases and nearly 30,000 deaths in the United States annually. The accurate staging of a patient with prostate cancer is critical for selection of appropriate treatment strategies, especially as applied to differentiating between those with localized or regional disease who can be treated with curative intent versus those with metastatic disease. Whether or not surgery, radiation, and systemic hormone and/or chemotherapy are appropriate for a given patient is driven in large part by the clinical stage.(1) According to the recent updated National Comprehensive Cancer Network guidelines, 99mTc-phosphonate bone scintigraphy (bone scan) and computed tomography (CT) or magnetic resonance imaging MRI scans remain the standard imaging modalities used for prostate cancer staging. However, bone and CT scans have demonstrated limited diagnostic accuracy in earlier disease settings $(2,3)$ which in turn limits accurate staging necessary for optimal prostate cancer management.

Accurate detection of metastatic disease is a particularly important goal because metastatic prostate cancer requires a different treatment approach and carries a significantly worse prognosis as compared to local disease. Positron Emission Tomography (PET) is a noninvasive technique that can image bone and soft tissue in a single modality, evaluate high-grade tumors that may not produce PSA, and provide a quantifiable data using the standardized uptake value (SUV). However, in prostate cancer PET tracers that image metabolic pathways, such as C-11 choline, C-11 acetate, and F18-deoxyglucose (FDG) suffer from suboptimal sensitivity and specificity in detection of

regional and distant metastatic disease. Recently, small ligands for PET-imaging have 
been developed which target the cell surface protein Prostate Specific Membrane Antigen (PSMA), which is overexpressed in prostate cancer cells, but also expressed to some extent in other organs and blood vessels.(4) The radiopharmaceuticals based on the PSMA ligands have demonstrated high diagnostic accuracy for the detection of both regional and distant metastatic prostate cancer. The proPSMA trial demonstrated that PSMA PET/CT has greater staging accuracy than conventional imaging consisting of bone scan and CT for initial staging of patients with high-risk prostate cancer.(5) This supports the use of a single PSMA PET/CT rather than two conventional imaging modalities in this setting.

Recent efforts in standardizing the assessment of PSMA scans have resulted in a number of PSMA PET evaluation and reporting systems, including PSMA-RADS, EANM, and Prostate Cancer Molecular Imaging Standardized Evaluation (PROMISE).(6-8) While all the proposed criteria are focused on the characterization of individual PSMA lesions based on the location and the definition of significant uptake, the PROMISE standard is also proposing a patient level staging (miTNM), which is based on the detection and location of the disease in the PSMA PET/CT image. A Recent study comparing such standardized assessment has shown that they have a high inter-reader reproducibility. $(9,10)$

However, the adoption and implementation of PROMISE criteria in routine clinical practice and investigational studies is limited by the fact that it must be done manually and is labor-intensive. The manual work can be greatly facilitated through automation by deep learning image analysis. The structural radiological processes including the segmentation of anatomical structures (from CT) can be automated to contextualize and 
characterize the functional imaging. The application of deep learning in automating the whole-body segmentation in PET/CT is the foundational framework of automating the PROMISE criteria. In this study, we introduce and evaluate the analysis of PSMA-PET images through aPROMISE, a deep learning platform to both automate standardized staging as well as generate a fully quantitative assessment of PSMA defined disease burden at the lesion and patient level.

\section{MATERIALS AND METHODS}

\section{Patient Population}

The purpose of our study was to evaluate the performance of the aPROMISE technology in standardizing the staging and quantification of prostate cancer disease. This investigation was a retrospective analysis of 109 Veterans with unfavorable intermediate and high-risk primary prostate cancer who underwent ${ }^{18} \mathrm{~F}-\mathrm{DCFPyL}$ PET/CT (PSMA) under clinical trial NCT03852654. NCT03825654 is a single arm trial of PSMA PET/CT in Veterans who also underwent conventional imaging with bone scan, CT or MRI. The study was approved by the local institutional review board at Veterans Affairs (VA) hospital (PCC 2018-100989), with waiver for individual informed consent.

\section{Study Design}

To validate the performance of aPROMISE, two independent board-certified nuclear medicine physicians -(three years of clinical experience) reviewed the PSMA images with the assistance of aPROMISE. No prior instructions were given, and the readers solely and independently relied on the aPROMISE workflow (detailed below). In summary, the 
aPROMISE provides the reader with automated segmentation and quantification of lesions with pre-selected miTNM type. The reader can choose to accept or override the aPROMISE automated selections at the level of each individual lesion. A final report is auto-generated based on the aPROMISE assisted read.

First, the aPROMISE-assisted staging was evaluated against the conventional image staging obtained from the routine clinical reports. Conventional imaging in every patient included: ${ }^{99 m}$ Tc-methyl diphosphonate bone scan or ${ }^{18} \mathrm{~F}$-Sodium Fluoride PET/CT $(\mathrm{NaF})$, and computed tomography $(\mathrm{CT})$ or magnetic resonance imaging (MRI) of the pelvis. Second, we evaluated the reproducibility of the staging and lesion quantification between the two independent aPROMISE-assisted reads. Finally, we evaluated the clinical association of quantitative PSMA uptake (miPSMA-index) with baseline clinical variables - Gleason Score, and PSA values. All patients were staged in four distinct categories:

1. Patients with localized disease and absence of regional lymph node or distant metastatic disease - miNOMO,

2. Patients with regional lymph node disease but absence of distant metastatic disease - miN1M0;

3. Patients with absence of regional lymph node but presence of distant metastatic disease - miN0M1;

4. Patients with presence of both regional lymph node and distant metastatic disease $-\operatorname{miN} 1 \mathrm{M} 1$. 


\section{aPROMISE and miPSMA index}

The aPROMISE v1.1 is a web-application, a class II software as a medical device (SaMD) developed by EXINI Diagnostics AB, Lund Sweden to standardize and quantify PSMA imaging in prostate cancer. aPROMISE is enabled with deep learning that automatically analyzes the CT image to segment anatomical regions in detail, including individual vertebrae, ribs, pelvic bones, and soft tissue organs including the prostate (Figure 1). The anatomical contextualization of the molecular image $(\mathrm{mi})$ is used to stage the patient based on location and extent of the disease in the primary prostate tumor (miT), local/regional pelvic lymph node disease (miN) and distant metastasis (miM). Subsequently, the PET image is analyzed to detect target lesions. aPROMISE technology enables implementation of standard guidelines like PROMISE in standardizing PSMA assessment.(6) Merging the target lesion information with the anatomical location, each target lesion is quantified in terms of both intensity and volume and summarized by tissue type to generate the miPSMA index. The aPROMISE report is created automatically with both aggregated information as well as detailed information on a per lesion basis. Manual controls are provided as fallback to augment automatic analysis.

In the PROMISE criteria, Eiber et al. defined the miPSMA score of a lesion as 0 when uptake is below aorta, 1 when uptake is between aorta and liver, 2 when uptake is between liver and the parotid gland, and 3 when uptake is above parotid gland. The miPSMA lesion index is a continuous extension of this, defined by linear interpolation from the lesion SUVmean and the aorta and liver SUV references as follows:

$$
\begin{gathered}
\text { LesionSUV }_{\text {mean }} \leq \text { AortaSUV }_{\text {ref }} \\
\text { LesionIndex }^{\text {LesionSUV }}=\frac{\text { mean }}{\text { AortaSUV }} \text { ref }
\end{gathered}
$$




$$
\begin{aligned}
& \text { AortaSUV }_{\text {ref }} \leq \text { LesionSUV }_{\text {mean }} \leq \text { LiverSUV }_{\text {ref }}: \\
& \text { LesionIndex }=1+\frac{\text { LesionSUV }_{\text {mean }}-\text { AortaSUV }_{\text {ref }}}{\text { AortaSUV }} \text { ref }
\end{aligned}
$$

$$
\begin{aligned}
& \text { LiverSUV }_{\text {ref }} \leq \text { LesionSUV }_{\text {mean }} \leq 2 \times \text { LiverSUV }_{\text {ref }} \text { : } \\
& \text { LesionIndex }=2+\frac{\text { LesionSUV }_{\text {mean }}-\text { LiverSUV }_{\text {ref }}}{\text { LiverSUV }_{\text {ref }}}
\end{aligned}
$$$$
2 \times \text { LiverSUV }_{\text {ref }} \leq \text { LesionSUV }_{\text {mean }}: \text { LesionIndex }=3
$$

The use of the parotid gland as a threshold has been replaced by 2 times liver reference since it is not certain that the parotid glands are included in all PSMA PET/CT scans. For each miTNM type, lesion uptake is aggregated into the Intensity-weighted Total Lesion uptake Volume (ITLV). This PSMA-index is defined as:

$$
I T L V_{\text {mix }}=\sum_{\text {Lesionoftypemix }} \text { LesionIndex } \times \text { LesionUptakeVolume }
$$

where miX can be any of miT, miN, miMa/b/c.

\section{Statistical Analysis}

Descriptive statistics were used to compare the aPROMISE staging to that of the conventional imaging. Cohen's pairwise kappa agreement was used to evaluate the interreader variability of aPROMISE-assisted staging - miNOM0, miN1M0, and miNOM1. Spearman and Kendall correlation coefficient was used to evaluate the inter-reader variability of the quantitative assessment (miPSMA-index) of each stage. Student's t-test 
was used to evaluate the miPSMA-index values (in tumor) in the risk groups defined by the PSA and the Gleason Score. All statistical analyses were carried out using R version 4.0.2.

\section{RESULTS}

109 consecutive patients were included in the analysis. Baseline characteristics of the patients are detailed in Table 1. Conventional Imaging staged 87 of the 109 patients as localized disease with no regional lymph node or distant metastasis (NOMO), 8 patients with regional lymph node disease (N1M0), 14 patients with metastatic disease without regional lymph node disease (NOM1), and no patients with both regional and distant metastatic disease (N1M1). Of the 14 patients identified as N0M1, all were conventionally staged with bone metastasis (NOM1b) by ${ }^{18} \mathrm{~F}$ Sodium Fluoride PET/CT scan and did not undergo ${ }^{99 m}$ Tc-methyl diphosphonate bone scan.

The duration of the aPROMISE assisted read, from selecting a patient to generating a complete report, was recorded at a mean of 3.2 minutes (range $1.8-5.1$ minutes) per scan for reader 1 and 3.4 minutes (range 2.3 to 5.8 minutes) for reader 2 . The comparative assessment of conventional against the aPROMISE assisted PSMA staging is detailed in Table $\mathbf{2 a}$ and Table $\mathbf{2 b}$. Both aPROMISE assisted PSMA analyses demonstrated significant upstaging in patients with localized and regional tumor burden and downstaging of patients that were positive for distant bone metastasis by $\mathrm{NaF}$ PET/CT. In aPROMISE-assisted read-1, of the 87 patients who were determined to be negative for local (N1) or distant (M1) metastatic disease by conventional imaging, 20 (23\%) were upstaged in PSMA imaging assessment to have regional lymph node $(\mathrm{N}=13)$ 
and distant metastatic disease $(\mathrm{N}=6)$. Similarly, of the 8 patients staged as having pelvic nodal local only disease (N1), $2(25 \%)$ were upstaged to having distant metastatic disease. Notably, a significant population with bone metastatic disease (by conventional imaging), were down staged by the aPROMISE-assisted PSMA imaging - 4/14 (29\%). Examples of the down staged aPROMISE-assisted PSMA read against the NaF is demonstrated in Figure 2. The aPROMISE-assisted read-2 had similar observations against the conventional imaging (Table $\mathbf{2 b}$ ).

Inter-observer reproducibility of aPROMISE-reads: A comparison of the two independent aPROMISE-assisted read is detailed in Table 3. The kappa agreement between the two aPROMISE-assisted reads was 0.82 for categorization of patients with miN0M0, 0.90 for patient with miN1M0, and 0.77 for patients with miN0M1b. Among all the stages, the relatively modest discrepancy in aPROMISE-associated reads was most notable for isolated low intensity bone lesions. The quantitative reproducibility of miPSMA index in the cases that were categorized the same in the two independent aPROMISEassisted reads, miN0M0 (N=66), miN1M0 (17), miNOM1(N=12), is illustrated in Figure 3. The spearman correlation was at $0.93,0.96$ and 0.97 , respectively.

aPROMISE miPSMA index: As a continuous variable, miPSMA index in the prostate $(\mathrm{miT})$ of all patients $(\mathrm{N}=109)$ was correlated with PSA values $(\mathrm{t}=0.30 ; \mathrm{p}<0.0001)$. Figure 4 shows the miPSMA index values in the prostate, stratified in risk groups defined by the PSA and separately by the Gleason Score. There was a significant difference in the PSMA index values of patients with PSA of $\leq 10 \mathrm{ng} / \mathrm{ml}$ (median=17.61; IQR $8.75-$ 44.63) compared to that observed in patients with $\geq 20 \mathrm{ng} / \mathrm{ml}$ (median=54.63; IQR 27.55 80.79), $p=0.05$. Similarly, the PSMA-index values of prostate tumors with Gleason of $3+3$ 
(median=19.45; IQR 9.97 - 23.54) was significantly lower compared to tumors with Gleason grade $\geq 4+3$ (median=32.74; IQR $15.38-54.63)$, $p=0.01$.

\section{DISCUSSION}

The aPROMISE-assisted independent staging and the quantitative assessments of total disease burden were found to be consistent and reproducible between readers. Integrating PSMA assessment tools in the clinical workflow could allow for automation to provide efficiency, consistency, and accuracy in the staging and quantification of PSMA PET/CT. This study also demonstrated that the aPROMISE-assisted reads for PSMA PET/CT detected significantly more regional and metastatic lesions that were suspicious of disease than identified by the conventional imaging.

The ability of PSMA imaging to detect a greater number of suspicious metastatic lesions in comparison to conventional bone scan or CT has been evident across multiple studies.(11-14) The frequency of upstaging in nodal and distant metastasis by PSMA $\mathrm{PET} / \mathrm{CT}$ compared to conventional imaging in this cohort of patients with intermediate and high-risk prostate cancer was in line with previous reports. Notably, the biological dimension of PSMA in evaluating suspected metastatic disease was particularly apparent when comparing findings from $\mathrm{NaF}$ to PSMA imaging. Of the 14 patients categorized as M1b through NaF scan, four (29\%) were called negative in aPROMISE-assisted reads of their respective PSMA scan. As a bone metabolic scan $\mathrm{NaF}$ scan is known to be susceptible to non-pathophysiological features in bone like trauma, degenerative changes, fibrous dysplasia, etc. Of these four patients with lesions called on $\mathrm{NaF}$ image but not PSMA PET/CT, two demonstrated what appear to be more likely benign lesions 
on PSMA PET/CT that were called positive on the corresponding NaF imaging. The other two patients with discordant findings in between NaF and PSMA imaging had suspicious appearing sclerotic bone lesions that were not picked up on the aPROMISE reads due to low lesion PSMA intensity. One of these two patients underwent curative intent radical prostatectomy and remains free of biochemical recurrence almost one-year post surgery without additional therapy. The other patients delayed treatment and instead underwent a repeat $\mathrm{NaF}$ imaging six months later that was without interval change in the bone lesion, however imaging demonstrated progression within soft tissue. In these two cases, clinical follow-up was more consistent with the PSMA PET staging rather than NaF imaging. A more comprehensive comparison of PSMA and NaF imaging is beyond the scope of this study but will be followed up in a separate analysis.

The inter-reader agreement on the interpretation of PSMA PET/CT has been evaluated mostly using ${ }^{68} \mathrm{Ga}-\mathrm{PSMA} 11 \mathrm{PET} / \mathrm{CT}$. Fendler et. al evaluated inter-reader agreements in 50 patients with primary disease and after biochemical recurrence and found agreement $\mathrm{k}=0.62$ (for primary tumor), 0.74 (for nodes), and 0.88 (for bone lesions).(15) In a more homogenous biochemically recurrent patient population consisting of patients with PSA levels up to $0.6 \mathrm{ng} / \mathrm{ml}$, Miksch et al. demonstrated $\mathrm{k}=0.76$ (for primary tumor), 0.73 (for nodes), and 0.58 (for bone lesions).(16) In one study, focused exclusively on 50 patients who underwent ${ }^{18} \mathrm{~F}$-DCFPyL PET, an intra-class correlation coefficient of 0.79 was derived for nodal disease.(17) Similarly, the manual reproducibility of following the PROMISE classification has been reviewed and reported by Toriihara, A et al. JNM 2020, which demonstrated a moderate inter-reader agreement (0.67) for miTNM classification in PSMA PET/CT.(9) The agreements between the aPROMISE- 
assisted reads in our study compare favorably against these prior evaluations (Cohen's Kappa agreement >0.75), with a notable quick reading time (mean 3.2 and 3.4 minutes per scan). Notably, one reader in our study had considerably more prior experience in interpretation of PSMA PET/CT than the other. Still, a high degree of agreement was noted. Readers in our study did not get any strict guidance on lesion detection nor formal training on PROMISE criteria. The findings may suggest that an aPROMISE- assisted read, which involves automated segmentation, localization and lesion pre-selection, may nudge readers towards a moderately high agreement irrespective of their prior experience. This hypothesis warrants a multi-center multi-reader study for validation.

Quantitative metrics of disease burden may further enhance prognostic and predictive power of imaging. Currently, the automated bone scan index (aBSI) is the only FDA cleared SaMD that has been prospectively validated in a registration study as a prognostic imaging biomarker for metastatic prostate cancer.(18) The STAMPEDE trial investigated addition of radiation to the primary in M1 patients. In a post-hoc analysis that used aBSI to assess disease burden, aBSI was predictive of response to prostate radiotherapy.(19) The aBSI employs a machine learning algorithm that pre-selects and segments the lesions in bone and automatically computes a quantitative total tumor burden in Tc99m planar bone scans.(20) In some sense, the miPSMA index for quantification of disease burden defined by PSMA PET/CT can be considered a threedimensional analog of aBSI.

However, the automated miPSMA index offers a far more comprehensive assessment of disease burden. The miPSMA index is a continuous extension of the miPSMA score proposed in the PROMISE criteria. Like the miPSMA score, the miPSMA 
index is the PSMA quantification of an individual lesion in relation to the mean uptake in reference organs. The result is a linear quantification of PSMA burden for each lesion that can be summarized by each tissue type $-\mathrm{miT}, \mathrm{miN}, \mathrm{miM}_{\mathrm{a} / \mathrm{b} / \mathrm{c}}$. Our study showed an association between miPSMA-Index in the primary tumor and both Gleason grade and PSA value. This is consistent with prior studies reporting PSMA expression in the primary tumor is associated with higher Gleason grade and recurrence risk. $(21,22)$ We hypothesize that miPSMA index may be useful for selecting patients for PSMA targeted radiotherapy, where current trials largely use qualitative assessments of PSMA expression as inclusion criteria. Moreover, there is a potential role for the miPSMA-index in conjunction with morphologic findings as a quantitative response assessment posttreatment.

The purpose of our hypothesis generating study was to evaluate the performance of the aPROMISE technology for subsequent prospective clinical investigations. The findings here enable future investigations to evaluate any additive benefits of aPROMISEassisted reads over manual reads of PSMA PET/CT and to evaluate enhanced diagnostic performance of PSMA PET/CT when employing the aPROMISE software. Our study was limited in the number of independent reads and in its retrospective design. Therefore, the findings and the hypothesis presented here should be validated in a prospectively designed multi-reader and multi-institutional study design. In addition, lesions selected by aPROMISE have not been histopathologically validated. However, high specificity of PSMA-PET has been demonstrated in several recent studies.

Despite these limitations, our study demonstrates the performance of aPROMISE in an independent assessment. The incorporation of aPROMISE and miPSMA index in 
subsequent clinical investigational can further explore its clinical context of use for prospective validation.

\section{CONCLUSION}

aPROMISE-assisted PSMA PET/CT reads generate detailed imaging reports at the whole patient and lesion level within minutes. aPROMISE-assisted reads of PSMA PET/CT upstages patients as compared to conventional imaging. Moreover, aPROMISEassisted reads may standardize PSMA evaluation and reduce inter-reader variability, even amongst readers of differing baseline levels of experience. Prospective studies and direct manual comparison studies are required to validate these findings. The miPSMA index is a quantitative measure of lesion volume and relative intensity, is associated with Gleason grade and PSA, and describes overall and tissue-specific tumor burden. Evaluation of the miPSMA index as an imaging biomarker of disease burden is warranted in order to assess prognostic value. 


\section{KEY POINTS}

\section{Question}

Can the aPROMISE platform generate a consistent and standardized evaluation of PSMA scans?

\section{Pertinent Findings}

The comparison of the two independent aPROMISE-assisted reads demonstrated a high kappa agreement in staging of patients in each present. As a continuous variable, miPSMA index in the prostate was associated with risk groups defined by the PSA values and Gleason Score.

\section{Implication for patient care}

aPROMISE-assisted reads may standardize PSMA evaluation and reduce inter-reader variability, even amongst readers of differing baseline levels of experience. The miPSMA index is a quantitative measure of lesion volume and relative intensity, is associated with Gleason grade and PSA, and describes overall and tissue-specific tumor burden.

Conflict of Interest Disclosures: Drs J. Brynlofsson, and K. Johnsson are employees of EXINI

Diagnostics AB. Drs. A. Anand and P. Borelli has received honorary support from EXINI Diagnostics AB. All other authors have no disclosures to declare.

Funding/Support: Supported by EXINI Diagnostics AB (a wholly owned subsidiary of Progenics Pharmaceuticals Inc, USA). Drs Nickols is a PCF Young Investigator. 


\section{Reference}

1. Mohler JL, Antonarakis ES, Armstrong AJ, et al. Prostate Cancer, Version 2.2019, NCCN Clinical Practice Guidelines in Oncology. J Natl Compr Canc Netw. 2019;17:479-505.

2. Abuzallouf S, Dayes I, Lukka H. Baseline staging of newly diagnosed prostate cancer: a summary of the literature. J Urol. 2004;171:2122-2127.

3. Golimbu M, Morales $\mathrm{P}, \mathrm{Al}-\mathrm{Ask}$ ari S, Shulman $\mathrm{Y}$. CAT scanning in staging of prostatic cancer. Urology. 1981;18:305-308.

4. Budaus L, Leyh-Bannurah SR, Salomon G, et al. Initial Experience of (68)Ga-PSMA PET/CT Imaging in High-risk Prostate Cancer Patients Prior to Radical Prostatectomy. Eur Urol. 2016;69:393-396.

5. Hofman MS, Lawrentschuk N, Francis RJ, et al. Prostate-specific membrane antigen PET-CT in patients with high-risk prostate cancer before curative-intent surgery or radiotherapy (proPSMA): a prospective, randomised, multicentre study. Lancet. 2020;395:1208-1216.

6. Eiber M, Herrmann K, Calais J, et al. Prostate Cancer Molecular Imaging Standardized Evaluation (PROMISE): Proposed miTNM Classification for the Interpretation of PSMA-Ligand PET/CT. J Nucl Med. 2018;59:469-478.

7. Fanti S, Minozzi S, Morigi JJ, et al. Development of standardized image interpretation for 68Ga-PSMA PET/CT to detect prostate cancer recurrent lesions. Eur J Nucl Med Mol Imaging. 2017;44:1622-1635.

8. Rowe SP, Pienta KJ, Pomper MG, Gorin MA. PSMA-RADS Version 1.0: A Step Towards Standardizing the Interpretation and Reporting of PSMA-targeted PET Imaging Studies. Eur Urol. 2018;73:485-487.

9. Toriihara A, Nobashi T, Baratto L, et al. Comparison of 3 Interpretation Criteria for (68)Ga-PSMA11 PET Based on Inter- and Intrareader Agreement. J Nucl Med. 2020;61:533539.

10. Demirci E, Akyel R, Caner B, et al. Interobserver and intraobserver agreement on prostate-specific membrane antigen PET/CT images according to the miTNM and PSMA-RADS criteria. Nucl Med Commun. 2020;41:759-767.

11. Calais J, Czernin J, Cao M, et al. (68)Ga-PSMA-11 PET/CT Mapping of Prostate Cancer Biochemical Recurrence After Radical Prostatectomy in 270 Patients with a PSA Level of Less Than $1.0 \mathrm{ng} / \mathrm{mL}$ : Impact on Salvage Radiotherapy Planning. J Nucl Med. 2018;59:230-237.

12. Calais J, Kishan AU, Cao M, et al. Potential Impact of (68)Ga-PSMA-11 PET/CT on the Planning of Definitive Radiation Therapy for Prostate Cancer. J Nucl Med. 2018;59:1714-1721.

13. Maurer T, Gschwend JE, Rauscher I, et al. Diagnostic Efficacy of (68)Gallium-PSMA Positron Emission Tomography Compared to Conventional Imaging for Lymph Node Staging of 130 Consecutive Patients with Intermediate to High Risk Prostate Cancer. J Urol. 2016;195:1436-1443. 
14. van Leeuwen PJ, Emmett L, Ho B, et al. Prospective evaluation of 68Gallium-prostatespecific membrane antigen positron emission tomography/computed tomography for preoperative lymph node staging in prostate cancer. BJU Int. 2017;119:209-215.

15. Fendler WP, Calais J, Allen-Auerbach M, et al. (68)Ga-PSMA-11 PET/CT Interobserver Agreement for Prostate Cancer Assessments: An International Multicenter Prospective Study. $J$ Nucl Med. 2017;58:1617-1623.

16. Miksch J, Bottke D, Krohn T, et al. Interobserver variability, detection rate, and lesion patterns of (68)Ga-PSMA-11-PET/CT in early-stage biochemical recurrence of prostate cancer after radical prostatectomy. Eur J Nucl Med Mol Imaging. 2020;47:2339-2347.

17. Werner RA, Bundschuh RA, Bundschuh L, et al. Interobserver Agreement for the Standardized Reporting System PSMA-RADS 1.0 on (18)F-DCFPyL PET/CT Imaging. J Nucl Med. 2018;59:1857-1864.

18. Armstrong AJ, Anand A, Edenbrandt L, et al. Phase 3 Assessment of the Automated Bone Scan Index as a Prognostic Imaging Biomarker of Overall Survival in Men With Metastatic Castration-Resistant Prostate Cancer: A Secondary Analysis of a Randomized Clinical Trial. JAMA Oncol. 2018;4:944-951.

19. Ali A, Hoyle AP, Parker CC, et al. The Automated Bone Scan Index as a Predictor of Response to Prostate Radiotherapy in Men with Newly Diagnosed Metastatic Prostate Cancer: An Exploratory Analysis of STAMPEDE's "M1|RT Comparison". Eur Urol Oncol. 2020;3:412419.

20. Ulmert D, Kaboteh R, Fox JJ, et al. A novel automated platform for quantifying the extent of skeletal tumour involvement in prostate cancer patients using the Bone Scan Index. Eur Urol. 2012;62:78-84.

21. Hupe MC, Philippi C, Roth D, et al. Expression of Prostate-Specific Membrane Antigen (PSMA) on Biopsies Is an Independent Risk Stratifier of Prostate Cancer Patients at Time of Initial Diagnosis. Front Oncol. 2018;8:623.

22. Demirci E, Kabasakal L, Sahin OE, et al. Can SUVmax values of Ga-68-PSMA PET/CT scan predict the clinically significant prostate cancer? Nucl Med Commun. 2019;40:86-91. 


\section{Tables}

Table 1. Patient Characteristics ( $\mathrm{N}=109)$

\begin{tabular}{|l|l|l|l|}
\hline Age & & Gleason Score & $\mathbf{N}(\%)$ \\
\hline average & 70 & $3+3$ & $13(7 \%)$ \\
\hline median & 69 & $3+4$ & $28(23 \%)$ \\
\hline minimum & 55 & $4+3$ & $24(18 \%)$ \\
\hline maximum & 86 & $\geq 4+4$ & $49(36 \%)$ \\
\hline Race & $\mathbf{N}(\%)$ & Diagnosis PSA & Value (ng/ml) \\
\hline White & $54(49 \%)$ & average & $20.4 \mathrm{ng} / \mathrm{mL}$ \\
\hline African American & $44(41 \%)$ & median & $13.55 \mathrm{ng} / \mathrm{mL}$ \\
\hline Hispanic & $7(7 \%)$ & minimum & $3.03 \mathrm{ng} / \mathrm{mL}$ \\
\hline Asian Pacific Islander & $3(2 \%)$ & maximum & $167.92 \mathrm{ng} / \mathrm{mL}$ \\
\hline Native American & $1(1 \%)$ & & \\
\hline Clinical T Stage & $\mathbf{N}(\%)$ & Percent of Positive Core & $\mathbf{N}(\%)$ \\
\hline cT1/2 & $62(57 \%)$ & $<25 \%$ & $18(17 \%)$ \\
\hline cT3 & $47(43 \%)$ & $25 \%-50 \%$ & $28(26 \%)$ \\
\hline & & $51 \%-75 \%$ & $14(13 \%)$ \\
\hline & & $>75 \%$ & $28(26 \%)$ \\
\hline & & Unknown & $11(10 \%)$ \\
\hline
\end{tabular}


Table 2a. aPROMISE-PSMA Staging read 1 vs. Conventional Imaging; N=109

\begin{tabular}{|c|c|c|c|c|c|}
\hline & & \multicolumn{4}{|c|}{$\begin{array}{c}\text { Local and Distant Metastatic Staging by } \\
\text { Conventional Imaging }\end{array}$} \\
\hline & & $\begin{array}{l}\text { NO M0 } \\
(\mathrm{N}=87)\end{array}$ & $\begin{array}{l}\mathrm{N} 1 \mathrm{M} 0 \\
(\mathrm{~N}=8)\end{array}$ & $\begin{array}{l}\text { N0 M1a/b } \\
(\mathrm{N}=14)\end{array}$ & $\begin{array}{l}\mathrm{N} 1 \mathrm{M} 1 \mathrm{a} / \mathrm{b} \\
(\mathrm{N}=0)\end{array}$ \\
\hline \multirow{4}{*}{$\begin{array}{l}\text { aPROMISE- } \\
\text { PSMA Staging } \\
\text { read } 1\end{array}$} & $\begin{array}{c}\operatorname{miNOM0} \\
(\mathrm{N}=71)\end{array}$ & 67 & 0 & 4 & 0 \\
\hline & $\begin{array}{c}\operatorname{miN} 1 \mathrm{M} 0 \\
(\mathrm{~N}=19)\end{array}$ & 13 & 6 & 0 & 0 \\
\hline & $\begin{array}{l}\operatorname{miN} 0 \mathrm{M} 1 \mathrm{a} / \mathrm{b} \\
\quad(\mathrm{N}=15)\end{array}$ & 6 & 0 & 9 & 0 \\
\hline & $\begin{array}{l}\operatorname{miN} 1 \mathrm{M} 1 \mathrm{a} / \mathrm{b} \\
\quad(\mathrm{N}=4)\end{array}$ & 1 & 2 & 1 & 0 \\
\hline
\end{tabular}

Table 2b. aPROMISE-PSMA Staging read 2 vs. Conventional Imaging; N=109

\begin{tabular}{|c|c|c|c|c|c|}
\hline & & \multicolumn{4}{|c|}{$\begin{array}{c}\text { Local and Distant Metastatic Staging by } \\
\text { Conventional Imaging }\end{array}$} \\
\hline & & $\begin{array}{c}\mathrm{N} 0 \mathrm{M} 0 \\
(\mathrm{~N}=87)\end{array}$ & $\begin{array}{c}\mathrm{N} 1 \mathrm{M} 0 \\
(\mathrm{~N}=8)\end{array}$ & $\begin{array}{c}\mathrm{N} 0 \mathrm{M} 1 \mathrm{a} / \mathrm{b} \\
(\mathrm{N}=14)\end{array}$ & $\begin{array}{c}\mathrm{N} 1 \mathrm{M} 1 \mathrm{a} / \mathrm{b} \\
(\mathrm{N}=0)\end{array}$ \\
\hline & $\begin{array}{c}\mathrm{miN0M0} \\
(\mathrm{N}=72)\end{array}$ & $\mathbf{6 8}$ & 0 & 4 & 0 \\
\cline { 2 - 6 } $\begin{array}{c}\text { aPROMISE- } \\
\text { PSMA } \\
\text { Staging } \\
\text { read 2 }\end{array}$ & $\begin{array}{c}\mathrm{miN1M0} \\
(\mathrm{N}=18)\end{array}$ & $\mathbf{1 2}$ & $\mathbf{6}$ & 0 & 0 \\
\cline { 2 - 6 } & $\begin{array}{c}\mathrm{miN} \text { (N)1a/b } \\
(\mathrm{N}=15)\end{array}$ & $\mathbf{6}$ & $\mathbf{0}$ & $\mathbf{9}$ & 0 \\
\cline { 2 - 6 } & $\begin{array}{c}\mathrm{miN} 1 \mathrm{M} 1 \mathrm{a} / \mathrm{b} \\
(\mathrm{N}=4)\end{array}$ & $\mathbf{1}$ & $\mathbf{2}$ & $\mathbf{1}$ & $\mathbf{0}$ \\
\hline
\end{tabular}


Table 3. aPROMISE-PSMA Read against aPROMISE-PSMA Read 2; N=109

\begin{tabular}{|c|c|c|c|c|c|}
\hline & & \multicolumn{4}{|c|}{$\begin{array}{l}\text { Local and Distant Metastatic Staging by } \\
\text { aPROMISE-PSMA Read } 1\end{array}$} \\
\hline & & $\begin{array}{c}\operatorname{miNOM0} \\
(\mathrm{N}=71)\end{array}$ & $\begin{array}{c}\operatorname{miN} 1 \mathrm{M0} 0 \\
(\mathrm{~N}=19)\end{array}$ & $\begin{array}{l}\mathrm{miNOM} 1 \mathrm{a} / \mathrm{b} \\
(\mathrm{N}=15)\end{array}$ & $\begin{array}{l}\operatorname{miN} 1 \mathrm{M} 1 \mathrm{a} / \mathrm{b} \\
\quad(\mathrm{N}=4)\end{array}$ \\
\hline \multirow{4}{*}{$\begin{array}{l}\text { aPROMISE- } \\
\text { PSMA } \\
\text { Read } 2\end{array}$} & $\begin{array}{c}\operatorname{miNOM0} \\
(\mathrm{N}=72)\end{array}$ & 67 & 2 & 3 & 0 \\
\hline & $\begin{array}{c}\operatorname{miN} 1 \mathrm{M} 0 \\
(\mathrm{~N}=18)\end{array}$ & 1 & 17 & 0 & 0 \\
\hline & $\begin{array}{l}\operatorname{miN0M1a/b} \\
(\mathrm{N}=15)\end{array}$ & 3 & 0 & 12 & 0 \\
\hline & $\begin{array}{c}\mathrm{miN} 1 \mathrm{M} 1 \mathrm{a} / \mathrm{b} \\
(\mathrm{N}=4)\end{array}$ & 0 & 0 & 0 & 4 \\
\hline
\end{tabular}




\section{Figures}
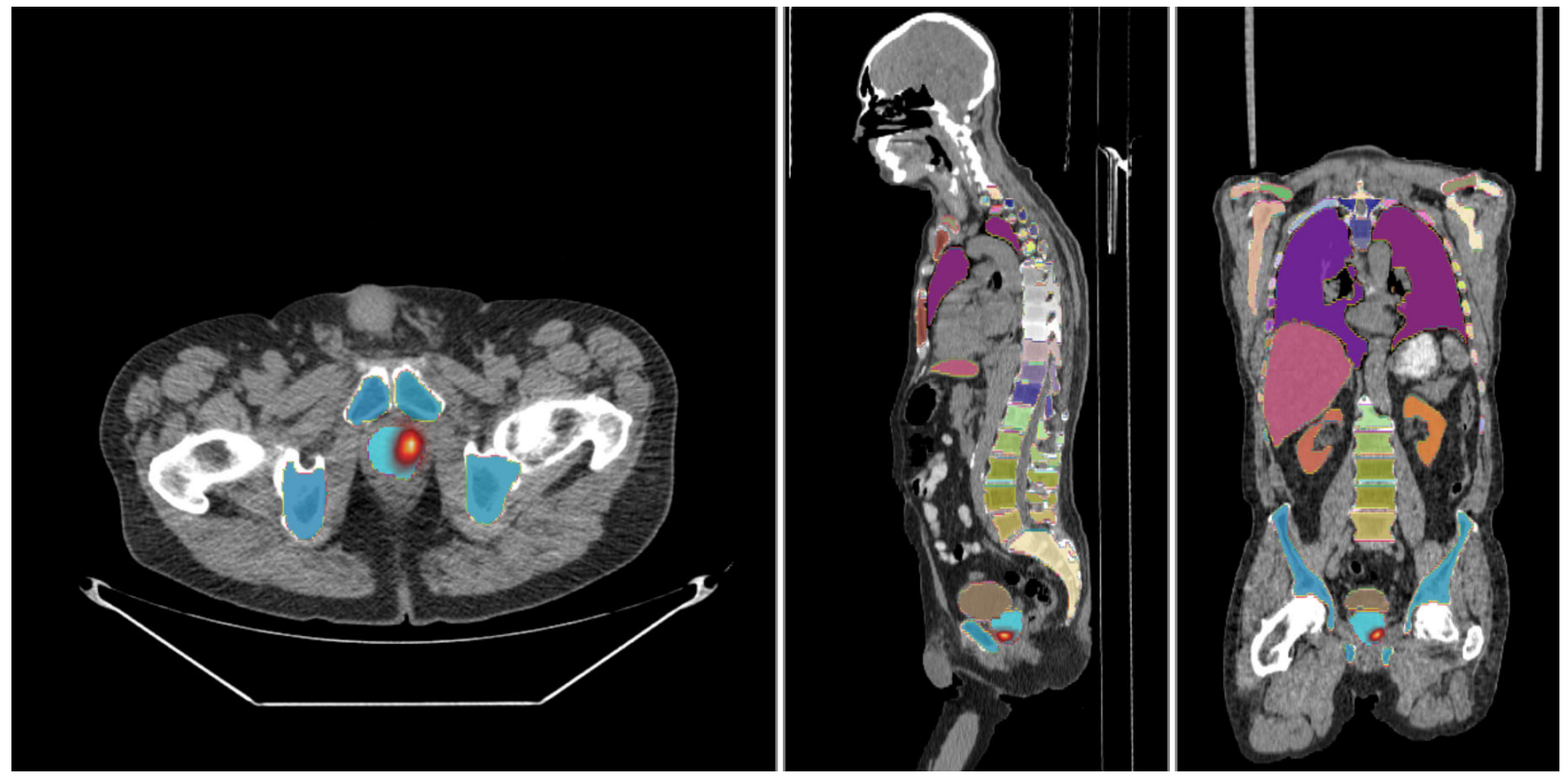

Figure 1. Deep learning enabled segmentation of anatomical context in low dose CT of PET/CT.

Individual color represents the respective segmented organ. The aPROMISE technology enables automated segmentation of reference organs, and anatomical delineation of the disease in the prostate (miT), regional lymph nodes (miN), and distance metastases (miM). 
A (Pt\# $\left.94{ }^{18} \mathrm{~F}-\mathrm{DCFPyL}\right)$
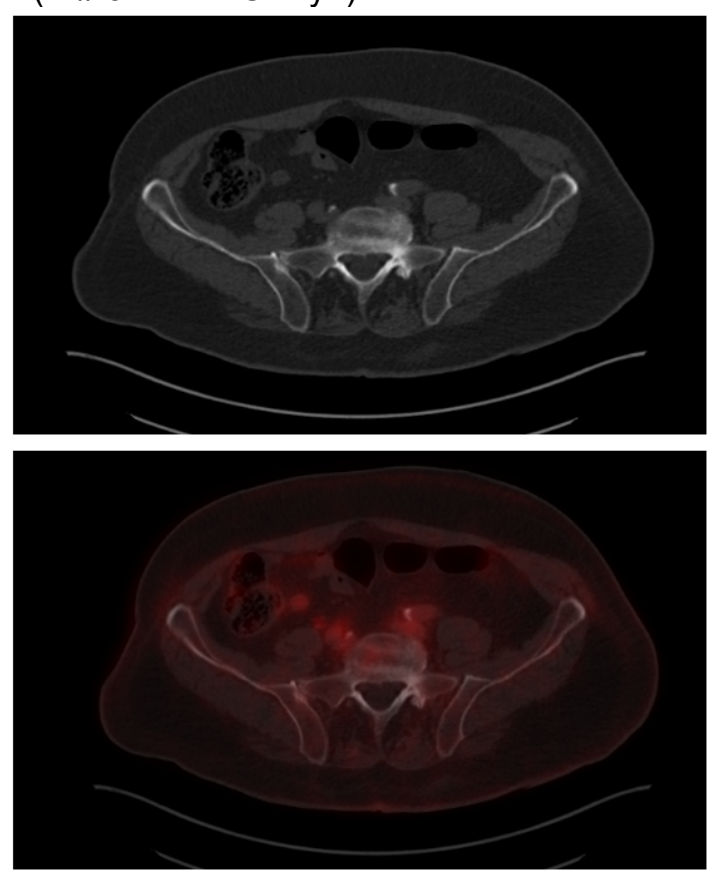

\section{C (Pt\# $\left.28{ }^{18} \mathrm{~F}-\mathrm{DCFPyL}\right)$}
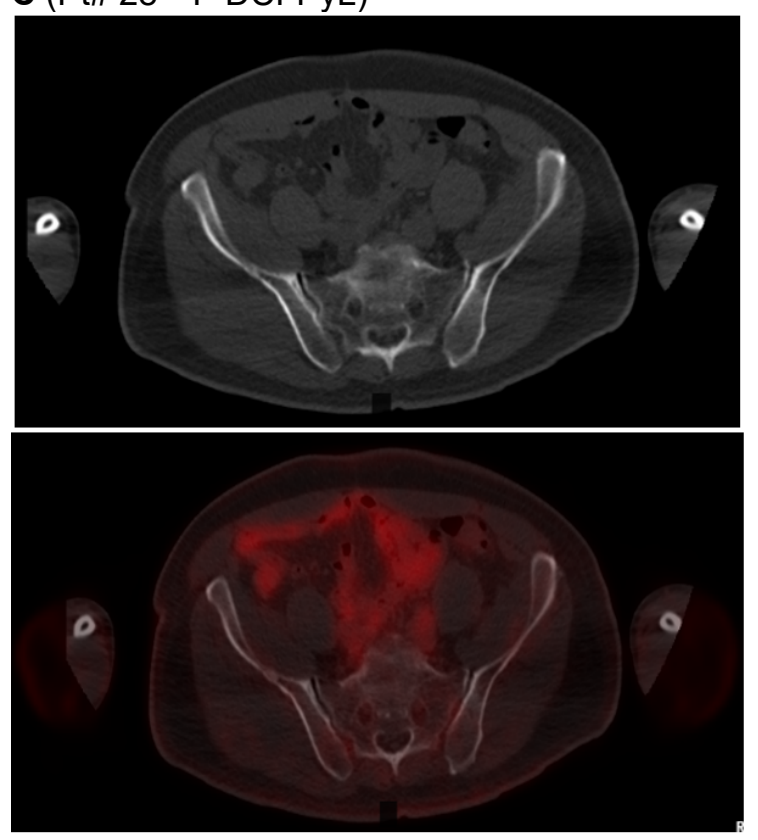

Figure 2. Example of cases (Pt\# 94 and Pt\#28) that were negative in the aPROMISE-assisted reads in ${ }^{18} \mathrm{~F}-\mathrm{DCFPyL}$

scan $\left(A\right.$ and $C$ ) compared to the $\mathrm{Na}^{18} \mathrm{~F}(\mathrm{~B}$ and $\mathrm{D})$ and were down staged from NOM1 to NOMO.

A
B ( Pt\# $\left.94 \mathrm{Na}^{18} \mathrm{~F}\right)$
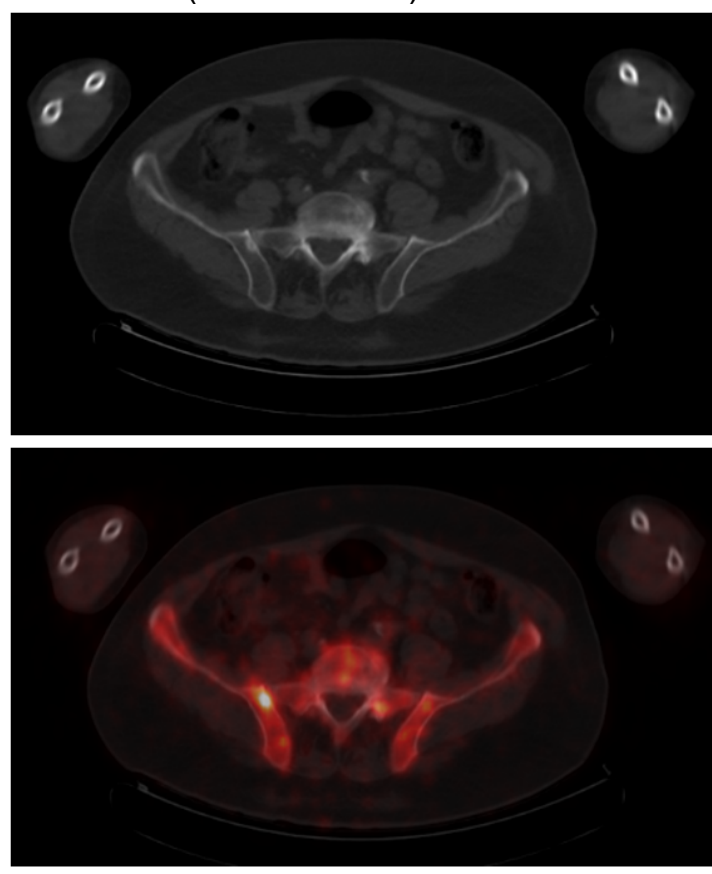

D $\left(\mathrm{Pt} \# 28 \mathrm{Na}^{18} \mathrm{~F}\right)$

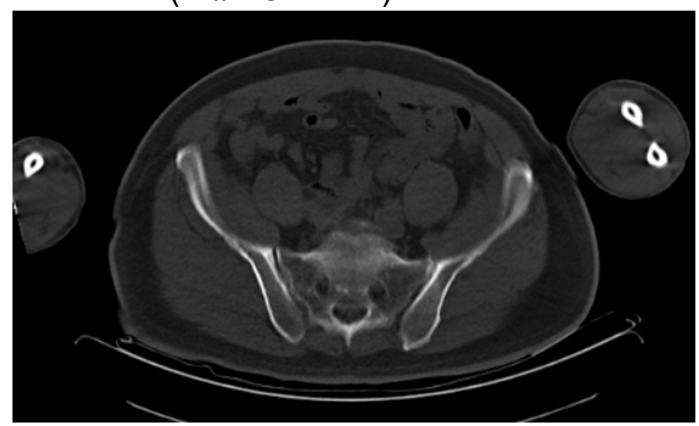

0

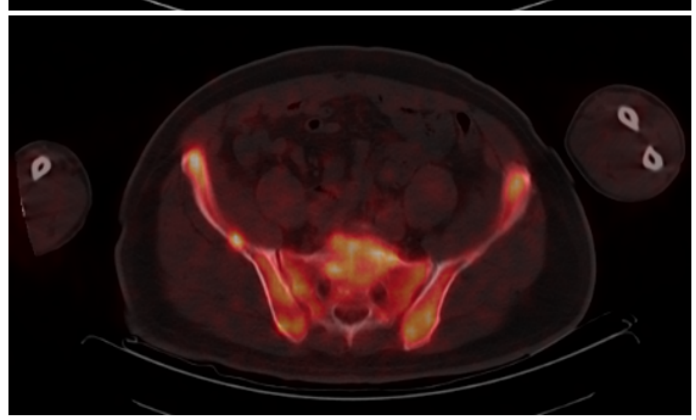




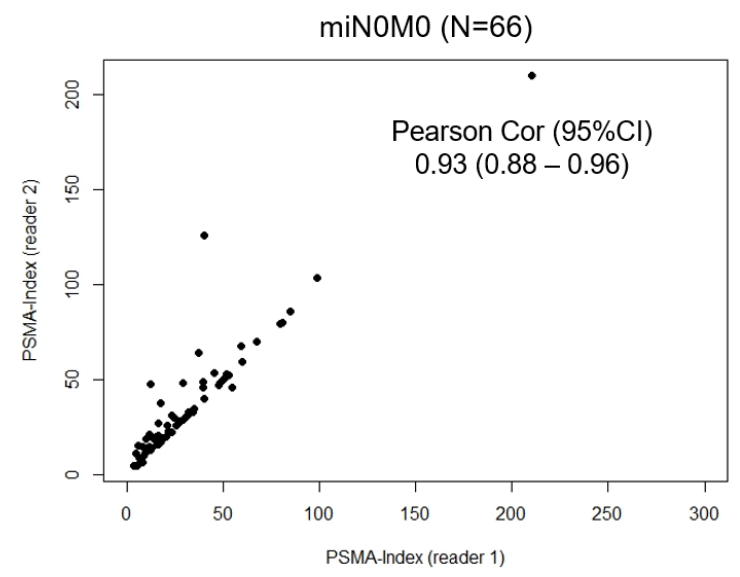

B

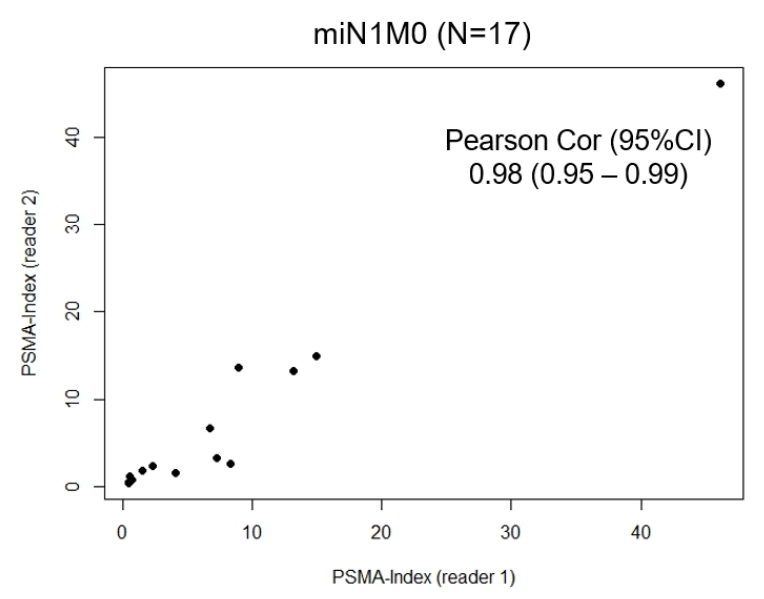

C

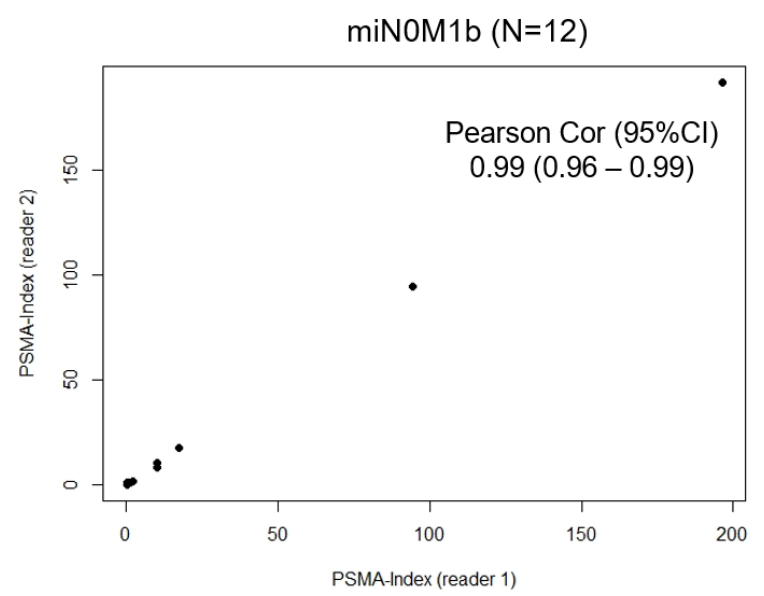

Figure 3. The quantitative reproducibility of the miPSMA-index in cases that were categorized the same in the two independent aPROMISE-assisted reads - miNOM0* (A), miN1M0 (B), and miNOM1(C). *One patient was excluded due to a manual segmentation error that incorporated the bladder. 
A.

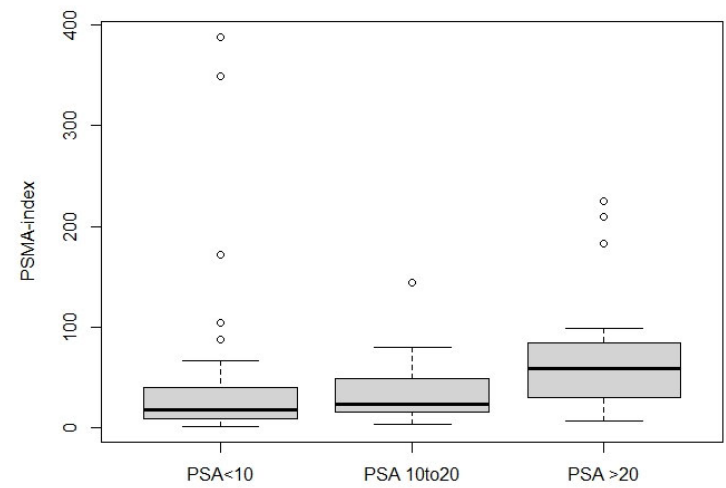

B.

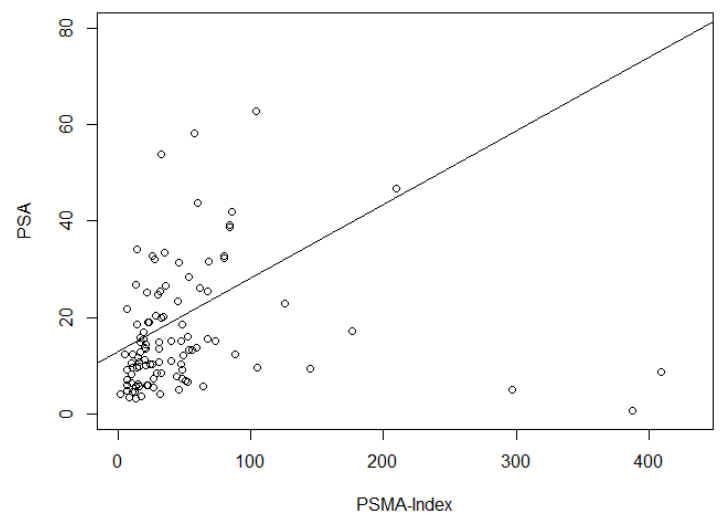

C.

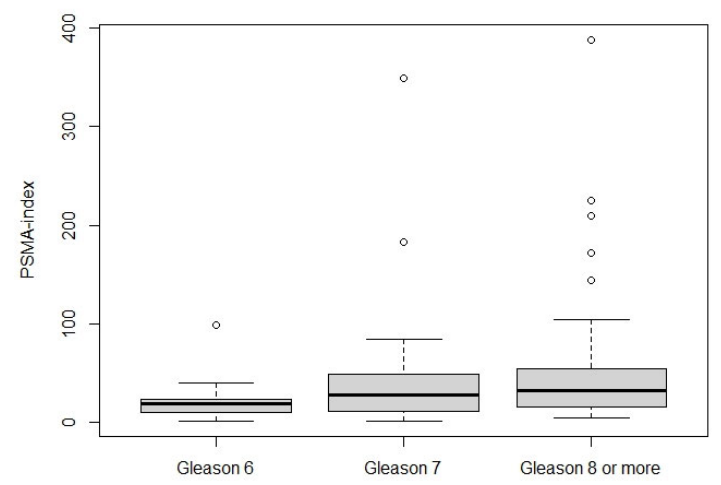

Figure 4. miPSMA-index values in the prostate, stratified by PSA (4A and $4 \mathrm{~B}$ ) and separately by Gleason grade (4C). 